\title{
Plataforma de Aprendizado Acessível da Dell (Dell Accessible Learning) Uma plataforma de ensino a distância acessível para todos
}

\author{
Marcos D. do Nascimento ${ }^{1}$, Pedro H. G. Muniz ${ }^{1}$, Lidiane C. Silva ${ }^{1}$, Eder F. Soares ${ }^{1}$, \\ Bruno da S. Queiroz ${ }^{1}$, Raquel F. Lima ${ }^{1}$, Nelson A. S. Lima ${ }^{1}$ \\ ${ }^{1}$ Centro de Pesquisa, Desenvolvimento e Inovação Dell (Dell Lead) \\ Av. Santos Dumont, 2456, Aldeota - Fortaleza - CE - Brazil \\ \{marcos.devaner, pedro.muniz, lidcastro, bruno, raquel, nelson\}@dellead.com \\ eder.soares@dell.com
}

\begin{abstract}
Research indicates that there is a significant number of people with disabilities in the world. These people find various accessibility barriers in their daily lives, professional training being an example. As a consequence, inserting people with disabilities in the labor market is a challenging task. This paper presents our work on an Accessible Distance Education Platform (Dell Accessible Learning), which was built on the basis of international accessibility guidelines and with the participation of people with disabilities in their design, implementation and validation. The use of the Dell Accessible Learning Platform has presented good results in what regards acceptance by the users and positive numbers in relation to the training of people with disabilities.
\end{abstract}

Resumo. Pesquisas indicam que há um número significativo de pessoas com deficiência no mundo. Essas pessoas encontram várias barreiras de acessibilidade em suas vidas diárias, treinamento profissional sendo um exemplo. Como consequência, inserir pessoas com deficiência no mercado de trabalho é uma tarefa desafiadora. Este artigo apresenta uma Plataforma de Ensino a Distância Acessivel (Dell Accessible Learning), que foi construída na base de diretrizes internacionais de acessibilidade e com a participação de pessoas com deficiência em seu desenho, implementação e validação. O uso da Plataforma Dell Accessible Learning tem apresentado bons resultados no que diz respeito a aceitação pelos usuários e números positivos em relação à formação de pessoas com deficiência.

\section{Introdução}

De acordo com o censo do Instituto Brasileiro de Geografia e Estatística de 2010, existem no Brasil cerca de 45 milhões de pessoas com deficiência (PcD) [Brasil 2010] ${ }^{1}$. E segundo estimativa da Organização Mundial da Saúde, mais de um bilhão de pessoas no mundo têm algum tipo de deficiência [World Health Organization 2011]. Essa parcela da população brasileira e mundial enfrenta dificuldades no acesso a educação formal e na

\footnotetext{
${ }^{1}$ Disponível em: ww2.ibge.gov.br/home/estatistica/populacao/censo2010/default.shtm. Acesso dia 5 de julho de 2019.
} 
VIII Congresso Brasileiro de Informática na Educação (CBIE 2019)

Anais dos Workshops do VIII Congresso Brasileiro de Informática na Educação (WCBIE 2019)

obtenção de formação profissional, já que, para essas pessoas, ainda há pouco planejamento no que concerne acessibilidade nesses e em outros domínios. Como consequência, sua inserção no mercado de trabalho é consideravelmente mais difícil do que a de pessoas sem deficiência, que em geral encontram menos barreiras de acessibilidade em atividades cotidianas ou laborais. Recursos tecnológicos podem auxiliar na melhoria do processo de aprendizagem das $\mathrm{PcD}$ e, consequentemente, na posterior absorção dessa força de trabalho. A importância de se encontrar soluções que ajudem a sanar esses problemas se mostra de forma ainda mais clara quando levamos em conta que, desde 1991, toda empresa no Brasil que conta com pelo menos 100 empregados deve obrigatoriamente preencher parte das vagas de emprego com PcD [Brasil 1991] $]^{2}$.

A Educação a distância se mostra como uma potencial grande aliada quando o objetivo é promover acessibilidade e inclusão na formação de pessoas com deficiência. Como essas pessoas muitas vezes têm dificuldade de se locomover e encontram outros tipos de barreiras em instituições de educação formal (cujos espaços físicos nem sempre são apropriados para recebê-las), realizar cursos de qualidade através de seus computadores pessoais (ou através de outros dispositivos com acesso à internet) é uma grande vantagem. Já no que diz respeito a absorção da força de trabalho das $\mathrm{PcD}$, a área de tecnologia da informação se revela especialmente promissora, já que a maior parte do trabalho pode ser feito sentado, não exigindo muita locomoção.

Tendo em vista esse cenário, a equipe do Centro de Pesquisa, Desenvolvimento e Inovação Dell (Dell Lead) em Fortaleza, em parceria com a Universidade Estadual do Ceará (UECE) e com o Instituto de Estudos, Pesquisas e Projetos da UECE (IEPRO), deu início ao projeto de desenvolvimento de uma plataforma de ensino à distância acessível. Também conhecida como "Dell Accessible Learning", hoje a nossa plataforma já está disponível através do site do Dell Lead $^{3}$ e oferece cursos profissionalizantes voltados para diversas áreas, mas com foco em Tecnologia da Informação, contando com certificação emitida pela Universidade Estadual do Ceará. Vamos falar um pouco mais sobre a nossa proposta, principalmente sobre os recursos que tornam nossa plataforma acessível.

\section{A Plataforma de Aprendizado Acessível da Dell}

Temos como principal meta oferecer formação de qualidade para pessoas com e sem deficiência, principalmente aqueles que não tiveram oportunidade de se inserir na educação formal e no mercado de trabalho. No momento, nossa plataforma de Aprendizado Acessível atende bem principalmente PcD que tenham baixa visão, cegos, surdos ou pessoas com problemas auditivos.

O acesso a plataforma é disponibilizado exclusivamente para os alunos matriculados nos cursos. Sendo feita a matrícula, os alunos podem acessar todos os conteúdos das aulas através de computadores conectados à Internet. De maneira geral, a disponibilização dos cursos se dá através de parcerias com instituições privadas, governamentais e do terceiro setor.

Alunos que são PcD contam com uma série de recursos de acessibilidade, como

\footnotetext{
${ }^{2}$ Disponível em: camara.leg.br/legin/fed/lei/1991/lei-8213-24-julho-1991-363650-normaatualizadapl.pdf. Acesso dia 5 de julho de 2019.

${ }^{3}$ Disponível através do portal do Lead http://leadfortaleza.com.br/, no botão "Entrar", no canto superior direito da tela.
} 
VIII Congresso Brasileiro de Informática na Educação (CBIE 2019)

Anais dos Workshops do VIII Congresso Brasileiro de Informática na Educação (WCBIE 2019)

alertas sonoros, um tour adaptado para cegos (que funciona com um leitor de tela instalado), audiodescrição, tradução de conteúdos para a língua brasileira de sinais de forma a beneficiar estudantes surdos, uma ferramenta para solicitar interpretação do correio e do fórum, um botão para ativar o alto contraste da página, botões para redimensionamento da fonte (que pode ter seu tamanho aumentado ou diminuído de acordo com a necessidade do usuário) e comandos de voz. Essas funcionalidades permitem que os alunos acessem os conteúdos de forma dinâmica e confortável, eliminando possíveis barreiras de acesso à informação. Pensando em eliminar tais barreiras, nossa plataforma se adequa as diretrizes de acessibilidade de conteúdos Web, estabelecida pelas Web Content Accessibility Guidelines (WCAG) ${ }^{4}$, documento reconhecido internacionalmente, mas que infelizmente ainda não é adotada por todos os desenvolvedores que criam sistemas para a Internet.

A plataforma disponibiliza cursos de capacitação profissional em diversas áreas, como Educação, Empreendedorismo e Vendas, Línguas Estrangeiras e Tecnologia da Informação. Os conteúdos estão disponíveis em português, inglês, língua brasileira de sinais (libras) e língua americana de sinais. No total, são 17 cursos: Introdução à Educação a Distância, Atendimento ao Cliente, Empreendedorismo e Ética, Inglês Básico, Customer Service (em inglês), Informatics (em inglês), Introdução à Lógica de Programação, Fundamentos de Programação Orientada a Objetos com Java, Introdução a Aplicativos On-line do Google, Informática Prática, Introdução ao Desenvolvimento de Aplicações em Java, Fundamentos de Banco de Dados com Java, Fundamentos de Desenvolvimento Móvel utilizando Android API e Java, Fundamentos de Programacao para a Web com Java, Padronização de Projetos Web com Java EE e Spring Framework, Planejamento e Desenvolvimento de Aplicações Android utilizando Google APIs e Integração de Aplicações e Serviços com Java. Todos os cursos têm certificação fornecida pela UECE. O principal objetivo da nossa plataforma EaD é facilitar o acesso à formação profissional de pessoas com deficiência e, ao mesmo tempo, apresentar uma solução para empresas que ainda precisam se encaixar nas exigências da lei brasileira de cotas. Dessa forma, atendemos uma necessidade do mercado e contribuímos para promover educação, cidadania e dignidade a comunidade de PcD no Brasil.

Na Figura 1 abaixo, podemos ver a página inicial da Plataforma. Esta é a primeira página a ser visualizada pelo usuário ao acessar a Dell Accessible Learning com seu login e senha. O usuário tem acesso imediato a todos os cursos nos quais está matriculado, que são listados em uma barra horizontal de rolagem. Caso o aluno esteja matriculado em mais de três cursos, os demais podem ser acessados através de setas dos dois lados da tela. Na tela inicial há também atalhos para as "Novidades" e para a Agenda do aluno. Em uma barra vertical à esquerda, temos atalhos para voltar ao Início, para acessar os Cursos, visualizar a Agenda do aluno, o Correio Interno, suas Notas e para o Glossário dos cursos, nesta ordem. Já na parte superior da tela, temos atalhos de navegação e o Menu de acessibilidade, com botões diversos que oferecem recursos para tornar a navegação na plataforma acessível.

A Figura 2 mostra os atalhos de acessibilidade disponíveis na Plataforma, que permitem navegar por toda a plataforma usando apenas atalhos de teclado ou comandos de voz. Eles são listados dentro de uma caixa de texto que aparece quando o usuário aciona

\footnotetext{
${ }^{4}$ Web Content Accessibility Guidelines 2.1, W3C World Wide Web Consortium Recommendation 05 June 2018 (Última versão disponível em: https://www.w3.org/TR/WCAG21/)
} 
VIII Congresso Brasileiro de Informática na Educação (CBIE 2019)

Anais dos Workshops do VIII Congresso Brasileiro de Informática na Educação (WCBIE 2019)

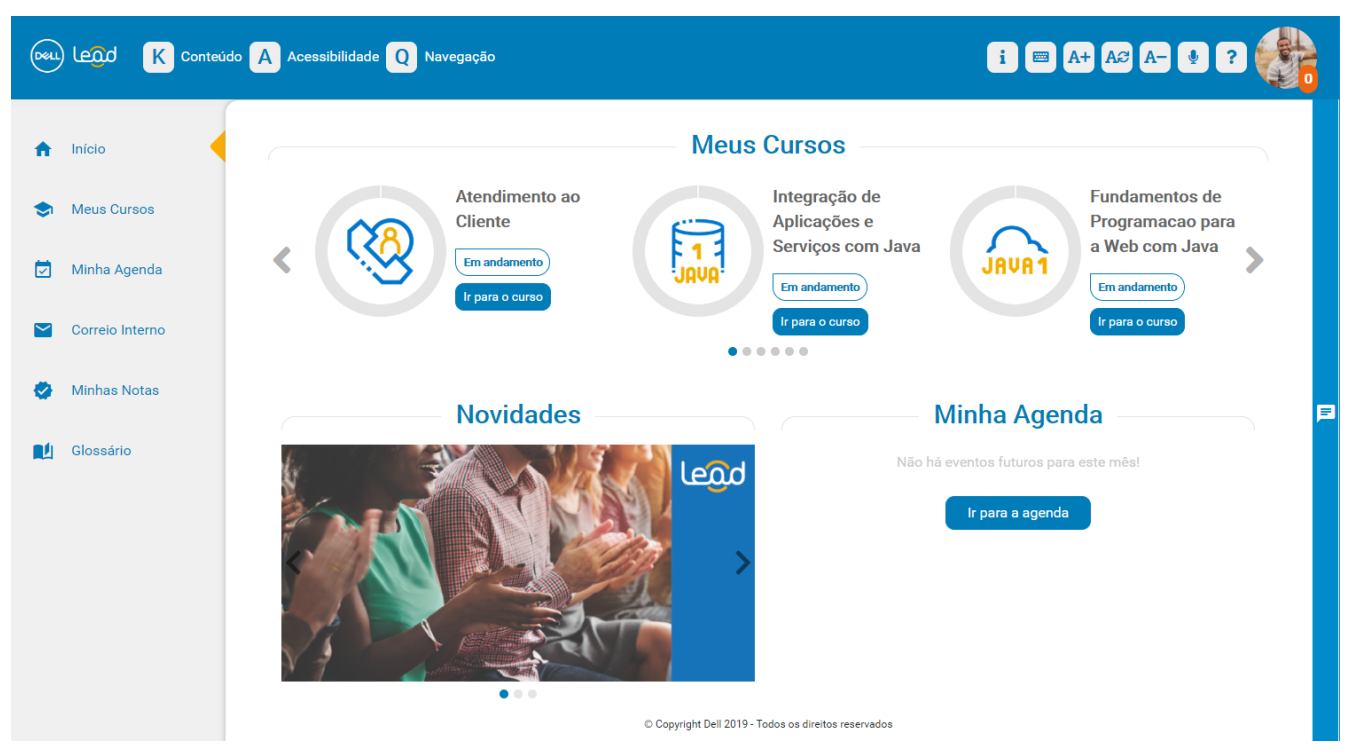

Figura 1. Página inicial da Plataforma Dell Accessible Learning

o botão marcado com um "i" no Menu de Acessibilidade, que é uma barra com botões de recursos de acessibilidade que está disponível em todas as páginas da plataforma, sempre aparecendo no canto superior direito. Por padrão, ele aparece como o menu representado pela Figura 3. Mais botões de acessibilidades podem ser ativados, dependendo da necessidade do usuário e do tipo de deficiência que ele possui. Na Figura 4, vemos todas as opções de recursos que podem ser habilitados para o Menu de Acessibilidade. Essas opções podem ser escolhidas pelo usuário ao acessar o seu perfil, disponível ao acionar o ícone que corresponde a sua imagem de perfil, no canto superior direito da tela. Na seção de editar seu perfil, além de poder editar informações pessoais, o aluno pode marcar ou desmarcar os botões que devem aparecer no menu de acessibilidade, salvando suas preferências para interações futuras com a plataforma.

Nas Figura 5 e 6 mais abaixo, podemos ver a aplicação de um dos recursos de acessibilidade disponíveis. Vemos nelas a primeira página da primeira lição do curso de Integração de Aplicações e Serviços com Java. Na Figura 5, vemos a página com o tamanho de fonte padrão. Na Figura 6 podemos ver a mesma tela com a fonte aumentada através do botão específico para esta finalidade no Menu de Acessibilidade. Perceba que a fonte de todo o site aumenta, inclusive os links e as palavras no menu, e que o layout da página não é prejudicado, acompanhando o aumento da fonte.

\section{Arquitetura e Módulos}

Tendo como base o OpenOlat ${ }^{5}$, a plataforma Dell Accessible Learning foi concebida e desenvolvida como um grande sistema distribuído [Tanenbaum et al. 1996]. Ela possui vários módulos que interagem entre si. Seguindo este conceito, os módulos são executados em diferentes servidores, evitando sobrecarga em um único servidor, garantindo maior escalabilidade para o sistema. A Figura 7 mostra esses diferentes módulos em servidores diferentes. A plataforma, como principal aplicação, consome os serviços de outras aplicações via webservice REST [Neumann et al. 2018]. O Openolat, por exem-

\footnotetext{
${ }^{5} \mathrm{https}: / /$ www.openolat.com
} 
VIII Congresso Brasileiro de Informática na Educação (CBIE 2019)

Anais dos Workshops do VIII Congresso Brasileiro de Informática na Educação (WCBIE 2019)

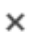

Teclas de atalho

\begin{tabular}{|c|c|}
\hline AltGr + A - Acessibilidade & Alt $\mathrm{Gr}+\mathrm{K}$ - Conteúdo \\
\hline AltGr + B - Contatos & AltGr + L - Lista de Aulas * \\
\hline AltGr + C - Cursos & AltGr +0 - Oficina * \\
\hline AltGr + E - Calendário & AltGr + P- Perfil \\
\hline AltGr + F - Fórum * & AltGr + Q - Navegação \\
\hline AltGr + G - Glossario & AltGr + R - Relatório de Notas \\
\hline AltGr + I - Email Interno & AltGr + S - Logout \\
\hline \multicolumn{2}{|c|}{ * Disponível somente no curso } \\
\hline \multicolumn{2}{|c|}{ Comandos de voz ** } \\
\hline Início & Glossario \\
\hline Meus Cursos & Aumentar Fonte \\
\hline Editar Perfil & Diminuir Fonte \\
\hline Agenda/Calendário & Resetar Fonte \\
\hline Correio & Sair \\
\hline Minhas Notas & Notificações \\
\hline
\end{tabular}

Figura 2. Caixa de texto com os atalhos de acessibilidade e comandos de voz para navegação na Plataforma

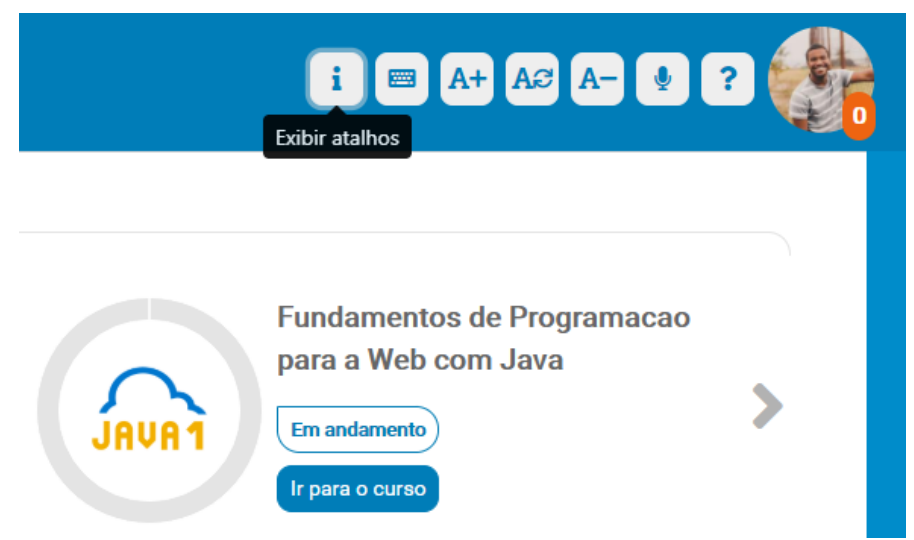

Figura 3. Detalhe do menu de acessibilidade com as funções padrões, no canto superior direito da tela

plo, é utilizado como repositório de cursos e materiais didáticos, além dos serviços para gerenciamento dos cursos.

Como mostrado na Figura 7, a plataforma de ensino a distância também se conecta via REST ao módulo de CHAT, que é executado em um servidor separado, para permitir que alunos e tutores falem uns aos outros em tempo real. O BigBlueButton ${ }^{6}$ é usado para videoconferência. Todas essas aplicações usam postgresql ${ }^{7}$ como o banco de

\footnotetext{
${ }^{6}$ https://bigbluebutton.org/

${ }^{7}$ https://www.elephantsql.com/docs/index.html
} 
VIII Congresso Brasileiro de Informática na Educação (CBIE 2019)

Anais dos Workshops do VIII Congresso Brasileiro de Informática na Educação (WCBIE 2019)

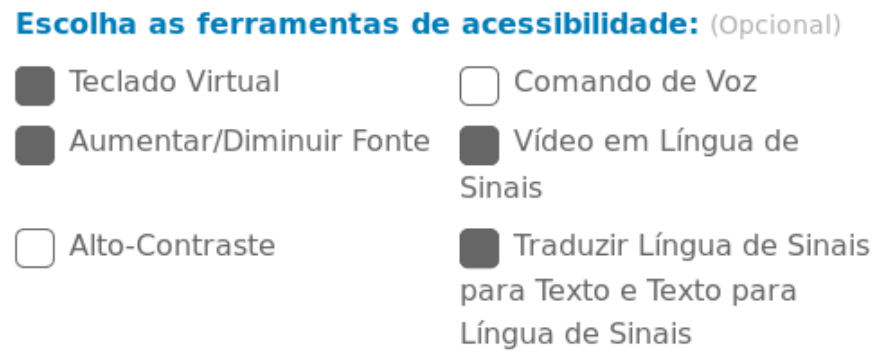

Alertas Sonoros

Figura 4. Opções acessibilidade disponíveis para marcação na seção "editar perfil" para personalizar o menu de acessibilidade

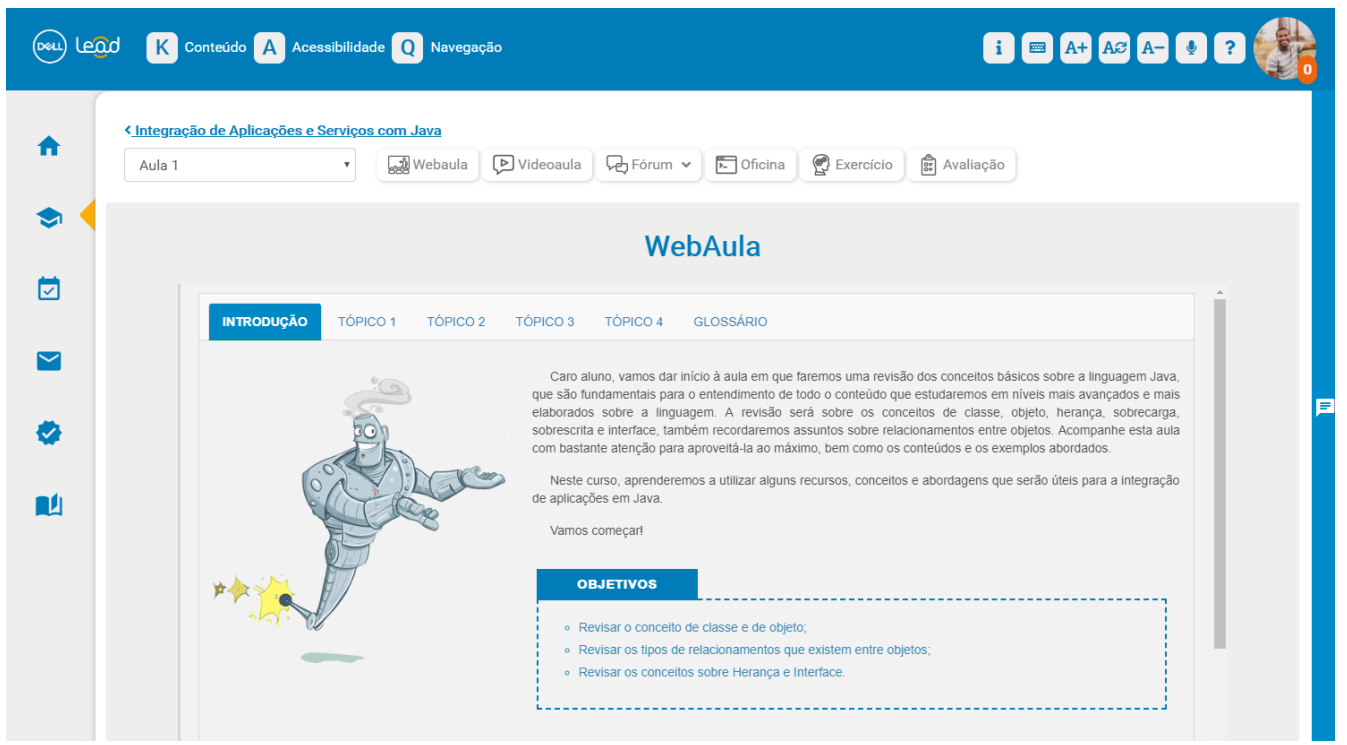

Figura 5. Primeira aula do curso de Integração de Aplicações e Serviços com Java, com tamanho de fonte padrão

dados e cada um tem seu próprio banco de dados. Embora a versão WEB seja responsiva, possibilitando seu uso por meio de dispositivos móveis, uma versão móvel da Dell Accessible Learning também foi desenvolvida. O aplicativo está disponível para dispositivos Android e iOS [Nascimento et al. 2017]. Sendo assim, a plataforma possibilita aos seus usuários/alunos maior flexibilidade e mobilidade para realização das atividades de aula.

\section{Processo de desenvolvimento}

O desenvolvimento da plataforma Dell Accessible Learning possui um processo iterativo composto por três etapas:

1. prototipação: é realizado um teste de interação com protótipos de tela. Assim, conseguimos validar a interação do usuário de acordo com o seu perfil, fazendo os ajustes necessários para cada perfil de usuário antesdo processo de desenvolvimento.

2. desenvolvimento: nesta etapa, implementa-se o código, além de aplicarem-se testes de funcionalidade e acessibilidade, verificando se existem erros ou proble- 
VIII Congresso Brasileiro de Informática na Educação (CBIE 2019)

Anais dos Workshops do VIII Congresso Brasileiro de Informática na Educação (WCBIE 2019)

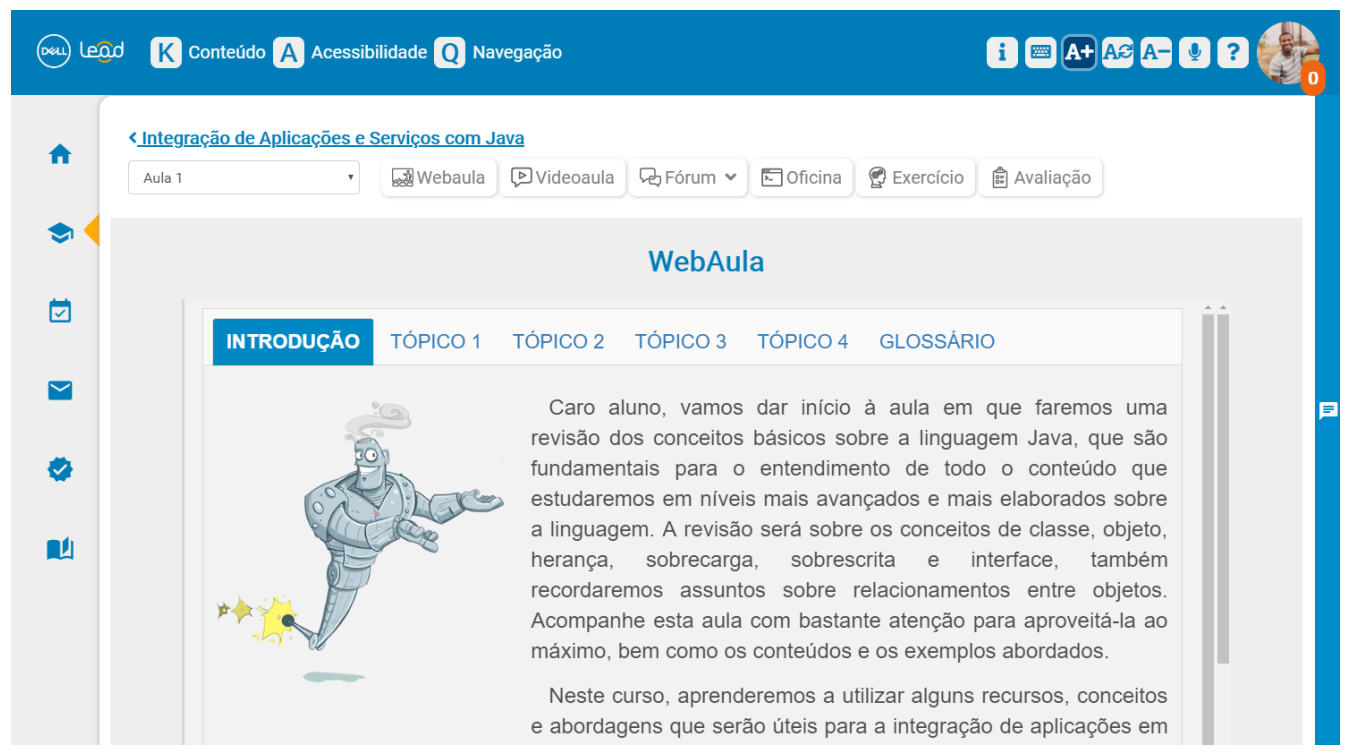

Figura 6. Primeira aula do curso de Integração de Aplicações e Serviços com Java, com tamanho de fonte aumentado quatro vezes

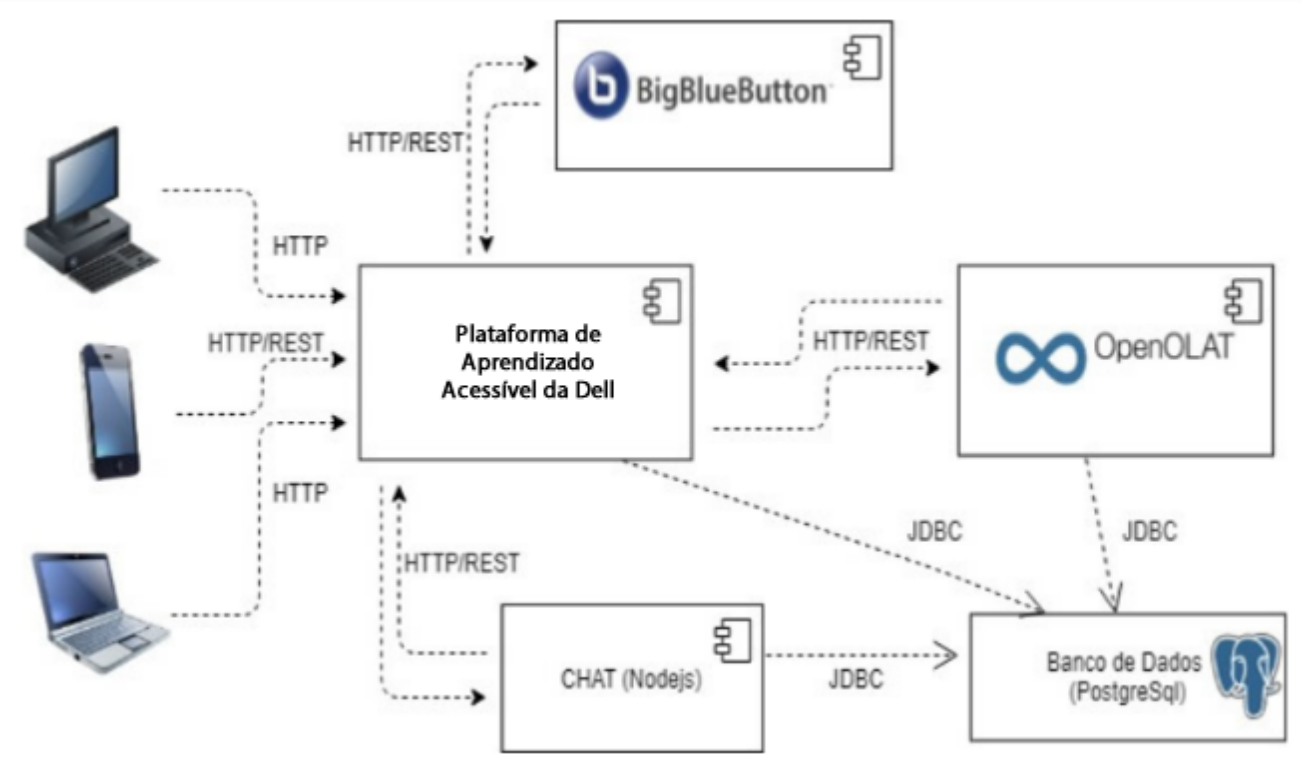

Figura 7. Arquitetura e módulo da Plataforma Dell Accessible Learning

mas de acessibilidade na estrutura do código. Os problemas de acessibilidade são verificados usando o "AccessMonitor", um validador automático que verifica a aplicação das diretrizes de acessibilidade, de acordo com as diretrizes de acessibilidade da WCAG 2.0 [Caldwell et al. 2008] no conteúdo HTML. Em caso de algum problema de funcionalidade, ele volta ao desenvolvimento para ajustes.

3. validação: A validação é realizada em ambiente de teste, e durante este processo os testadores PcD e não-PcD validam o módulo integrado com a plataforma. Esta validação é feita através de testes de usabilidade. Finalmente, após a validação, o módulo é integrado à versão final do usuário. 
VIII Congresso Brasileiro de Informática na Educação (CBIE 2019)

Anais dos Workshops do VIII Congresso Brasileiro de Informática na Educação (WCBIE 2019)

\section{Considerações finais}

Tanto a nossa plataforma web quanto o aplicativo para Android desenvolvido para acompanhá-la estão em constante evolução. Visamos sempre torná-la cada vez mais acessível, buscando desenvolver novas tecnologias assistivas para ajudar $\mathrm{PcD}$ que querem obter a formação oferecida (tanto soluções de software quanto de hardware, que estão em desenvolvimento no nosso centro de pesquisa). Nossa equipe pedagógica está também trabalhando na criação de novos cursos e na tradução dos cursos existentes e da plataforma como um todo para outras línguas, a fim de beneficiar não só a população brasileira.

O feedback dos usuários sobre problemas de uso e todas as suas sugestões de melhorias são catalogadas por uma equipe de suporte, que as encaminha para os devidos responsáveis, de forma a sempre atualizar o sistema. Estamos trabalhando para que a plataforma e o App atendam cada vez mais uma gama maior de pessoas com deficiência, realizando pesquisas para levantamento de requisitos específicos para cada deficiência ainda não atendida. Além da manutenção e evolução das atuais versões da Dell Accessible Learning para navegador e Android, também estamos desenvolvendo uma versão híbrida para que usuários de outros sistemas operacionais tenham acesso ao nosso conteúdo.

Todos os nossos esforços têm como objetivo atender e motivar pessoas com e sem deficiência a encontrarem seu próprio espaço na sociedade. Contribuímos para isso tentando lhes oferecer um ambiente de aprendizado que seja o mais agradável, interativo, intuitivo e acessível possível. Acreditamos que através de ferramentas como a nossa plataforma, pessoas com deficiência, que em geral têm suas necessidades negligenciadas, podem ser empoderadas, tendo acesso a novas oportunidades de aprendizado e, consequentemente, possibilidades de maior atuação na sociedade.

\section{Referências}

Brasil (1991). Lei no 8.213, de 24 de julho de 1991.

Brasil (2010). Censo do Instituto Brasileiro de Geografia e Estatística. IBGE.

Caldwell, B., Cooper, M., Reid, L. G., and Vanderheiden, G. (2008). Web content accessibility guidelines (wcag) 2.0. WWW Consortium (W3C).

Nascimento, M. D., Queiroz, B., Guimaraes, M., Silva, L. C., Soares, E., Oliveira, F., Ribeiro, D., and Ferreira, C. (2017). Aprendizado acessível. In Anais dos Workshops do Congresso Brasileiro de Informática na Educação, volume 6, page 110.

Neumann, A., Laranjeiro, N., and Bernardino, J. (2018). An analysis of public rest web service apis. IEEE Transactions on Services Computing.

Tanenbaum, A. S., Guerrero, G., and Velasco, Ó. A. P. (1996). Sistemas operativos distribuidos. Number QA76. 76063. T35. 3 1996. Prentice Hall México.

World Health Organization, W. H. O. (2011). World Report on Disability. WHO Library Cataloguing-in-Publication Data. 